\title{
SYMMETRIC ALGEBRAS OVER RINGS AND FIELDS
}

\author{
THOMAS C. CRAVEN ${ }^{\bowtie}$ and TARA L. SMITH
}

(Received 3 July 2013; accepted 10 July 2013; first published online 6 September 2013)

\begin{abstract}
Connections between annihilators and ideals in Frobenius and symmetric algebras are used to provide a new proof of a result of Nakayama on quotient algebras, and an application is given to central symmetric algebras.
\end{abstract}

2010 Mathematics subject classification: primary 16D99; secondary 15 A63.

Keywords and phrases: symmetric algebra, Frobenius.

\section{Introduction}

Throughout this paper, $R$ will denote a commutative ring with identity element 1 and $A$ will denote an associative unital $R$-algebra which is a finitely generated projective $R$-module. For any algebra $A$, the centre of $A$ will be denoted by $Z(A)$.

Definition 1.1. The algebra $A$ is called a Frobenius algebra if it is finitely generated and projective as an $R$-module and there exists a left $A$-module isomorphism $\varphi: A \cong A^{*}$, where $A^{*}$ denotes the ring $\operatorname{hom}_{R}(A, R)$ as a right $R$-module. Note that $A^{*}$ is an $(A, A)$-bimodule via $(a \cdot \lambda)(b)=\lambda(b a)$ and $(\lambda \cdot a)(b)=\lambda(a b)$ for any $a, b \in A, \lambda \in A^{*}$. If there exists a two-sided $A$-module isomorphism $\varphi: A \cong A^{*}$, then $A$ is called symmetric $[1,3]$.

There has been a resurgence of interest in Frobenius algebras in recent years due to applications in coding theory (see, for example, $[6,7]$ ). In this note we consider the connections between hyperplanes and ideals in Frobenius and symmetric algebras over commutative rings. This allows us to develop a succinct, coordinate-free proof of a result of Nakayama [4] that determines when the quotient of a symmetric algebra over a field is again symmetric. As a corollary, we show the class of central symmetric algebras is identical to the class of central simple algebras.

The work of the second author was partially supported by the NSF IR/D program while working at the Foundation. However, any opinions, findings, and conclusions or recommendations expressed in this material are those of the author and do not necessarily reflect the views of the National Science Foundation.

(c) 2013 Australian Mathematical Publishing Association Inc. 0004-9727/2013 \$16.00 
Definition 1.2. A form $f$ on $A$ is a bilinear mapping $f: A \times A \rightarrow R$. The form is called associative if $f(a c, b)=f(a, c b)$ for all $a, b, c \in A$. It is called right nonsingular if $b \mapsto f(\cdot, b)$ is an $R$-module isomorphism from $A$ to $A^{*}$ and left nonsingular if $a \mapsto f(a, \cdot)$ is an $R$-module isomorphism. When both conditions hold, we say the form is nonsingular.

REMARK 1.3. Some references will refer to such forms as 'nondegenerate'. We shall need a distinction between this and a weaker condition also called nondegenerate, so we shall follow the convention in [5]. The weaker condition states that $f$ is nondegenerate if $f(a, b)=0$ for all $a \in A$ implies $b=0$ and $f(a, b)=0$ for all $b \in A$ implies $a=0$. Over a field these conditions are easily seen to be equivalent using a dimension argument.

Proposition 1.4. A finitely generated projective R-algebra $A$ is Frobenius if and only if there exists a nonsingular associative bilinear form $f: A \times A \rightarrow R$, and is symmetric if and only if there exists such a form which is also symmetric.

Proof. Assume first that $A$ is a Frobenius algebra. Since $A$ is Frobenius there exists a left $A$-module isomorphism $\varphi: A \cong A^{*}$. We define $f(a, b)=\varphi(b)(a)$. The form $f$ is right nonsingular since $\varphi$ is an isomorphism. Left nonsingularity holds for the same reason applied to the transpose mapping $\varphi^{\prime}$ defined in [2, page 2]. Since $f(a, c b)=\varphi(c b)(a)=[c \varphi(b)](a)=\varphi(b)(a c)=f(a c, b)$, we see that $f$ is associative.

For the converse, assume there is an associative nonsingular form $f$. Define $\varphi: A \rightarrow A^{*}$ by $\varphi(b)(a)=f(a, b)$. Then, by definition, $\varphi$ is an isomorphism as desired.

If $\varphi$ is a two-sided $A$-module isomorphism, then $f(a, b)=\varphi(b)(a)=(b \cdot \varphi(1))(a)=$ $\varphi(1)(a b)$ using the left module isomorphism, and $f(b, a)=\varphi(a)(b)=(\varphi(1) \cdot a)(b)=$ $\varphi(1)(a b)$ using the right module isomorphism, so $f(a, b)=f(b, a)$. Conversely, if such a form is symmetric, the left module structure of $\varphi: A \cong A^{*}$ is straightforward from associativity and the right module structure is shown by $(\varphi(b) \cdot a)(x)=\varphi(b)(a x)=$ $f(a x, b)=f(b, a x)=f(b a, x)=f(x, b a)=\varphi(b a)(x)$ for all $a, b, x \in A$.

\section{Hyperplanes and ideals}

We begin by defining the notion of a 'hyperplane' with respect to an associative bilinear form on an $R$-algebra $A$. In the case where $A$ is an algebra over a field, this notion coincides with the usual notion of a hyperplane as a subspace of codimension one. We then determine the maximal left and right ideals in a hyperplane. We conclude by exploring the connections between annihilators of ideals and intersections of hyperplanes. In particular, we see why results for fields only partially generalise due to different versions of the nondegeneracy hypothesis for forms on a module over a ring.

Definition 2.1. Given an $R$-algebra $A$ with associative bilinear form $f$, we can associate two (not necessarily distinct) hyperplanes to each nonzero $c \in A$. A (left) 
hyperplane with respect to $f$ is a set

$$
{ }_{c} H=\{x \in A: f(x, c)=0\} \quad(c \neq 0, c \in A),
$$

and a (right) hyperplane with respect to $f$ is a set

$$
H_{c}=\{x \in A: f(c, x)=0\} \quad(c \neq 0, c \in A) .
$$

Hyperplanes are $R$-submodules of $A$. If the form $f$ is symmetric, then ${ }_{c} H=H_{c}$ for all $c \in A$. In general, ${ }_{1} H=H_{1}$ since associativity implies $f(1, a)=f(a, 1)$ for all $a \in A$.

More generally, if $S \subseteq A$, we may define $H_{S}=\{x \in A: f(s, x)=0 \forall s \in S\}$. The set ${ }_{S} H$ is defined similarly. These are intersections of hyperplanes. If $S$ is a right ideal in $A$, then $H_{S}$ will be a left ideal, and if $S$ is a left ideal, then ${ }_{S} H$ will be a right ideal.

Definition 2.2. The hyperplane $H_{c}$ (respectively, ${ }_{c} H$ ) is nondegenerate if it contains no nontrivial left (respectively, right) ideals. It is symmetric if it contains all commutators of $A$.

Proposition 2.3. If an R-algebra $A$ is Frobenius, then A admits an associative bilinear form $f$ having a pair of nondegenerate hyperplanes ${ }_{c} H$ and $H_{c}$, for some $c \in A$. If the algebra $A$ is symmetric, then $A$ admits an associative bilinear form $f$ having a pair of symmetric nondegenerate hyperplanes ${ }_{c} H$ and $H_{c}$, for some $c \in Z(A)$.

Proof. Let $f$ be an associative nonsingular bilinear form as guaranteed by Proposition 1.4. Consider the hyperplane $H_{1}$. If $A x \subseteq H_{1}$, we have $0=f(1, A x)=$ $f(A, x)$ by associativity, whence $x=0$ since $f$ is nonsingular. Thus $H_{1}$ contains no nontrivial left ideals. Also $x A \subseteq{ }_{1} H$ implies that $0=f(x A, 1)=f(x, A)$, so that $x=0$. Therefore ${ }_{1} H=H_{1}$ is nondegenerate.

Now assume that $A$ is a symmetric algebra and again consider $H_{1}$. Then

$$
f(1, y x-x y)=f(1, y x)-f(1, x y)=f(y, x)-f(x, y)=0
$$

since $f$ is symmetric, and so $H_{1}$ is symmetric.

As the following proposition shows, the condition $c \in Z(A)$ is inextricably linked to the condition that $H_{c}$ (or $\left.{ }_{c} H\right)$ is symmetric.

Proposition 2.4. Let $A$ be a symmetric R-algebra, $f$ a nonsingular associative symmetric form on $A$ and $c \in A$. Then $H_{c}$ or ${ }_{c} H$ is symmetric if and only if $c \in Z(A)$.

Proof. For all $x, y \in A$,

$$
\begin{aligned}
f(c, y x-x y)=0 & \Longleftrightarrow f(c, y x)=f(c, x y) \\
& \Longleftrightarrow f(c y, x)=f(x y, c)=f(x, y c)=f(y c, x) \\
& \Longleftrightarrow f(c y-y c, x)=0 \\
& \Longleftrightarrow c y-y c=0 \quad(\forall y \in A) \\
& \Longleftrightarrow c \in Z(A) .
\end{aligned}
$$

The proof for ${ }_{c} H$ is analogous. 
Notice that the proof of Proposition 2.3 did not use the full force of nonsingularity, but rather only nondegeneracy of the form $f$. We next obtain partial converses to the statements in Proposition 2.3; these are not full converses when nondegeneracy is not equivalent to nonsingularity.

Proposition 2.5. Let $A$ be an R-algebra with an associative bilinear form $f$. Let $H_{c}$ and ${ }_{c} H$ be a pair of nondegenerate hyperplanes with respect to $f$, for some $c \in A$. Then $f$ is nondegenerate. If the hyperplanes are also symmetric and $c \in Z(A)$, then there exists an associative nondegenerate symmetric form on A.

Proof. If $x \in A$ is such that $f(a, x)=0$ for all $a \in A$, then $f(c, A x)=f(c A, x)=0$; since $H_{c}$ is nondegenerate, $A x=0$, so $x=0$, which is to say that $b \mapsto f(\cdot, b)$ is injective. Also if $x \in A$ is such that $f(x, a)=0$ for all $a \in A$, then $f(x A, c)=f(x, A c)=0$; since ${ }_{c} H$ is nondegenerate, $x A=0$, so $x=0$. Thus the form $f$ is nondegenerate (but may not be nonsingular).

Now assume that the nondegenerate hyperplanes $H_{c}$ and ${ }_{c} H$ are also symmetric and $c \in Z(A)$. Then $f$ is nondegenerate as above. We first check that these hyperplanes are equal. Since $c \in Z(A)$, we have $f(x, c)=0$ if and only if $f(x c, 1)=f(c x, 1)=$ $f(c, x)=0$, and so we obtain ${ }_{c} H=H_{c}$. For all $x, y \in A$, define $g(x, y)=f(x, y c)$. Then $g$ is also associative. We next check that $g$ is nondegenerate. If $g(x, y)=0$ for all $y \in A$, then $x A \subseteq H_{c}$ and $x=0$ since $H_{c}={ }_{c} H$ is nondegenerate. Similarly, if $g(x, y)=0$ for all $x \in A$, then $A y \subseteq{ }_{c} H=H_{c}$ and so $y=0$. Finally, we must check that $g$ is symmetric. Since $H_{c}$ is symmetric, we have $f(x y-y x, c)=0$, from which we obtain $f(x y, c)=f(y x, c)$, or $f(x, y c)=f(y, x c)$. Now by definition of $g$, we have $g(x, y)=g(y, x)$ and thus $g$ is symmetric.

ExAmple 2.6. As an example where nondegeneracy is weaker than nonsingularity, we let $R=\mathbb{Z}$ and $A$ be the group ring $\mathbb{Z}\left[\mathbb{Z}_{2}\right]=\mathbb{Z} \oplus \mathbb{Z} g$, where $g^{2}=1$. Using $\{1, g\}$ as a basis, the form given by the matrix $\left(\begin{array}{ll}1 & 2 \\ 2 & 3\end{array}\right)$ is nonsingular since its determinant is a unit and so it induces an isomorphism with $A^{*}$. On the other hand, the form given by the matrix $\left(\begin{array}{ll}2 & 2 \\ 2 & 3\end{array}\right)$ is nondegenerate since its determinant is 2, a nonunit (but also nonzero), just as it is if viewed as a form over $\mathbb{Q}$. But it is not nonsingular. Specifically, the linear functional $\psi(x+y g)=x$ is not in the image of the mapping induced by the form $f$ since $f\left(x+y g, b_{1}+b_{2} g\right)=2 b_{1} x+2 b_{2} x+2 b_{1} y+3 b_{2} y$ cannot equal $x$ for any choice of $b_{1}, b_{2} \in \mathbb{Z}$.

Corollary 2.7. For a field $K$, a K-algebra $A$ is Frobenius if and only if $A$ admits an associative bilinear form $f$ having a pair of nondegenerate hyperplanes ${ }_{c} \mathrm{H}$ and $\mathrm{H}_{c}$, for some $c \in A$. The algebra $A$ is symmetric if and only $A$ admits an associative bilinear form $f$ having a pair of symmetric nondegenerate hyperplanes ${ }_{c} H$ and $H_{c}$, for some $c \in Z(A)$.

LEMMA 2.8. Let $A$ be an R-algebra with a nondegenerate associative bilinear form $f$ and let $c \in A$. The hyperplane $H_{c}$ contains $H_{c A}$ as its largest left ideal. Assume further that $f$ is symmetric. Then $H_{c A}$ is nontrivial if and only if $c$ is a (right) zero divisor. 
Proof. Suppose $J$ is a left ideal contained in $H_{c}=\{x: f(c, x)=0\}$. For all $a \in A$, we have $f(c, a J)=f(c, J)=0$, so $f(c A, J)=0$ and $J \subseteq H_{c A}$. Since $H_{c A}$ is itself a left ideal, it is the largest one.

Suppose now that $f$ is symmetric and $0 \neq x \in H_{c A}$. Then for all $a \in A$, we have $f(c a, x)=0$, which implies $f(x c, a)=f(x, c a)=0$ since $f$ is symmetric. By nondegeneracy, we obtain $x c=0$ and $c$ is a right zero divisor. Conversely, if $b c=0$ with $b \neq 0$, then for any $a \in A, f(a, b c)=0$, which implies $f(A b, c)=0$, so that $A b \subseteq H_{c}$. As above, this left ideal is contained in $H_{c A}$, showing that $H_{c A}$ is nontrivial.

Let $J$ be a two-sided ideal in a Frobenius algebra $A$. By associativity, we can write $f(x, J)=f(x J, A)$, so that, using the nondegeneracy of $f$, we have $x J=0$ if and only if $f(x, J)=0$. Similarly, $J x=0$ if and only if $f(J, x)=0$. Thus we can use the form $f$ to express the right annihilator $\mathrm{r}(J)$ and left annihilator $1(J)$ of $J$ as follows:

$$
\begin{aligned}
& \mathrm{r}(J)=\{x \in A: f(J, x)=0\}=H_{J}, \\
& \mathrm{l}(J)=\{x \in A: f(x, J)=0\}={ }_{J} H .
\end{aligned}
$$

These will play a crucial role in determining when a quotient of a symmetric algebra over a field is again symmetric.

Lemma 2.9. Let $J$ be a two-sided ideal in a symmetric algebra A. Then the left and right annihilators are equal; that is, $\mathrm{l}(J)=\mathrm{r}(J)$.

Proof. We have $x \in \operatorname{r}(J)$ if and only if $f(J, x)=0$ if and only if $f(x, J)=0$ (by symmetry) if and only if $x \in 1(J)$.

\section{Quotients of algebras}

We ask under what conditions a quotient of a symmetric algebra over a field is Frobenius or symmetric. This was done by Nakayama in the 1930s using matrix arguments [4], but we present a coordinate-free approach here.

Throughout this section, we shall assume that $A$ is a Frobenius algebra over a field $K$ with nondegenerate associative bilinear form $f$. We again point out that nonsingularity and nondegeneracy are equivalent concepts when the base ring is a field.

Lemma 3.1. Let $H$ be a maximal proper $K$-submodule of $A$. Then $H$ is a right hyperplane $H_{c}$ for some $c \in A$. Conversely, any right hyperplane $H_{c}$ is a maximal proper submodule. The analogous result holds for left hyperplanes as well.

Proof. Let $H$ be a maximal proper $K$-submodule of $A$ and set $H^{\perp}=\{x \in A$ : $f(x, H)=0\}$. Choose $0 \neq c \in H^{\perp}$. Then $A \neq H_{c}=\{x \in A: f(c, x)=0\} \supseteq H$. By maximality of $H$, we have $H_{c}=H$. Conversely, let $y \notin H_{c}$. Then $H_{c}+K y=A$ since given any $\alpha \in A$, there exists $r \in K$ such that $\alpha-r y \in H_{c}$. Specifically, if $r=f(c, \alpha) / f(c, y)$, then $f(c, \alpha-r y)=0$. Therefore $H_{c}$ is maximal.

Theorem 3.2. Let A be a symmetric K-algebra and let $J$ be a two-sided ideal of $A$. Then $A / J$ is Frobenius if and only if $\mathrm{r}(J)$ is a principal ideal generated by some $c \in A$, where $A c=c A$. This quotient is also symmetric if and only if $\mathrm{r}(J)=c A$ where $c \in Z(A)$. 
Proof. Suppose first that $\bar{A}=A / J$ is a Frobenius algebra. Let $\bar{H}$ be a nondegenerate hyperplane in $\bar{A}$. Let $H^{\prime}=\{x \in A: \bar{x} \in \bar{H}\}$. Then $H^{\prime}$ is a hyperplane in $A$, for if there exists a proper submodule $\tilde{H}$ properly containing $H^{\prime}$, then $\tilde{H} / J$ would be a proper submodule of $\bar{A}$ properly containing $\bar{H}$, contradicting the maximality of $\bar{H}$. We claim that $J$ is the largest left or right ideal in $H^{\prime}$. If not, assume that $I$ is a (left or right) ideal in $H^{\prime}$ not contained in $J$. Then $I+J \subseteq H^{\prime}$ so we may assume that $I$ properly contains $J$. But then $I / J$ is a nonzero (left or right) ideal in $\bar{H}$, contradicting the nondegeneracy of $\bar{H}$. Now $H^{\prime}=H_{c}$ for some $c \in A$ by Lemma 3.1, and by Lemma 2.8, this implies that $J=H_{c A}$. From this it follows that $J=r(c A)$. But since $A$ is symmetric, we have $J=\{x \in A: f(x, c A)=0\}$ as well, and so also $J=1(c A)$. Then we know that we have $\mathrm{r}(\mathrm{l}(c A))=c A=\mathrm{r}(J)$ since a Frobenius algebra over a Frobenius ring is a quasi-Frobenius ring [2, Corollary 20]. Since $\mathrm{r}(J)$ is a two-sided ideal, we have $A c \subseteq A c A=c A$. By an analogous argument, the left annihilator $1(J)$ is the principal left ideal $A c$, which is also two-sided, giving the reverse inclusion. Therefore $A c=c A$.

Conversely, assume that $\mathrm{r}(J)=1(J)=A c=c A$. Let $f$ be the associative, nondegenerate, symmetric bilinear form on $A$ given by Proposition 1.4. Define $\bar{f}: \bar{A} \times \bar{A} \rightarrow K$ by

$$
\bar{f}(\bar{x}, \bar{y})=f(x y, c)=f(x, y c) .
$$

To see that $\bar{f}$ is well defined, let $\tilde{x}=x+j_{1} \in x+J, \tilde{y}=y+j_{2} \in y+J$. Then

$$
f(\tilde{x} \tilde{y}, c)=f\left(\left(x+j_{1}\right)\left(y+j_{2}\right), c\right)=f(x y, c)+f\left(j_{1} y, c\right)+f\left(x j_{2}, c\right)+f\left(j_{1} j_{2}, c\right) .
$$

Since $c$ annihilates the two-sided ideal $J$ on both the right and left, and $j_{1}, x j_{2}, j_{1} j_{2}$ are all in $J$, we have $f(\tilde{x} \tilde{y}, c)=f(x y, c)$ as desired.

To complete the proof that $\bar{A}$ is Frobenius, we check that $\bar{f}$ is nondegenerate. Indeed,

$$
\begin{aligned}
\bar{f}(\bar{x}, \bar{y})=0 \forall \bar{y} & \Longleftrightarrow f(x, y c)=0 \forall y \\
& \Longleftrightarrow x \in H_{A c}=J \Longleftrightarrow \bar{x}=0
\end{aligned}
$$

and

$$
\begin{aligned}
\bar{f}(\bar{x}, \bar{y})=0 \forall \bar{x} & \Longleftrightarrow f(x, y c)=0 \forall x \\
& \Longleftrightarrow y c=0 \Longleftrightarrow y \in H_{c A}=J \Longleftrightarrow \bar{y}=0 .
\end{aligned}
$$

Finally, we have that $\bar{A}$ is symmetric if and only if $\bar{f}(\bar{x}, \bar{y})=\bar{f}(\bar{y}, \bar{x})$. We note that

$$
\begin{aligned}
\bar{f}(\bar{x}, \bar{y})=\bar{f}(\bar{y}, \bar{x}) \forall x \forall y & \Longleftrightarrow f(x y, c)=f(y x, c) \forall x \forall y \\
& \Longleftrightarrow x y-y x \in H_{c} \forall x \forall y \\
& \Longleftrightarrow c \in Z(A) \quad \text { by Proposition 2.4. }
\end{aligned}
$$

Now the proof is complete.

This theorem then allows the following characterisation of symmetric $K$-algebras $A$, where $K$ is a field, and $Z(A)=K$. To the best of the authors' knowledge, this result has not previously been observed. 
COROLlary 3.3. Central symmetric algebras over a field are simple.

Proof. By Theorem 3.2, if $A$ is a symmetric algebra and $J$ is an ideal of $A$ such that $A / J$ is symmetric, then $\mathrm{r}(J)=1(J)=A c$ for some $c \in Z(A)$. Let $J=\operatorname{rad}(A)$. Then if $A$ is an artinian $K$-algebra, $A / J$ is a semisimple $K$-algebra, so is necessarily symmetric. Combining these two facts, we see that if $A$ is central symmetric, then $\mathrm{r}(J)=1(J)=A c$ for some $c \in K$. In particular, $\mathrm{r}(J)=1(J)=0$ or $A$. Then we must have $\mathrm{r}(J)=A$, since $1(\mathrm{r}(J))=J$, and $\mathrm{l}(0)=A$, and of course $J \neq A$.

Thus $A$ is semisimple, so we may write $A$ as a direct sum of simple $K$-algebras, $A=\bigoplus M_{n_{i}}\left(D_{i}\right)$, where the $D_{i}$ are $K$-division algebras. The centre of $A$ is $\bigoplus Z\left(D_{i}\right) \cong$ $K$, so $A \cong M_{n}(D)$ where $D$ is a $K$-central division algebra.

EXAMPLE 3.4. The results of this section do not generally hold for rings since, as pointed out in Example 2.6, a nondegenerate form need not be nonsingular. For example, we can generalise the previous example by taking $R=\mathbb{Z}$ and $A$ to be the group ring $\mathbb{Z}\left[\mathbb{Z}_{2} \times \mathbb{Z}_{2}\right]$, where the copies of $\mathbb{Z}_{2}$ are generated by $g$ and $h$. Let $J$ be the ideal generated by $(1, h)$, so that $A / J$ is isomorphic to the algebra of Example 2.6. To make the proof of Theorem 3.2 work, we need to check that the induced bilinear form $\bar{f}$ is nonsingular (that is, it has a determinant which is a unit). It is easy to make examples where this does or does not happen, depending on the given symmetric form on $A$, but this does allow some application of the results of Section 2 outside of the field case.

\title{
References
}

[1] C. W. Curtis and I. Reiner, Representation Theory of Finite Groups and Associative Algebras (Interscience, New York, 1962).

[2] S. Eilenberg and T. Nakayama, 'On the dimension of modules and algebras II. Frobenius algebras and quasi-Frobenius rings', Nagoya Math. J. 9 (1955), 1-16.

[3] T. Y. Lam, Lectures on Modules and Rings (Springer, New York, 1999).

[4] T. Nakayama, 'On Frobeniusean algebras. I', Ann. of Math. (2) 40 (1939), 611-633.

[5] V. L. Popov (originator), 'Bilinear mapping', Encyclopedia of Mathematics, http://www.encyclopediaofmath.org/index.php?title=Bilinear_mapping\&oldid=13044.

[6] J. A. Wood, 'Duality for modules over finite rings and applications to coding theory', Amer. J. Math. 121 (1999), 555-575.

[7] J. A. Wood, 'Anti-isomorphisms, character modules and self-dual codes over non-commutative rings', Int. J. Inf. Coding Theory 1(4) (2010), 429-444.

\section{THOMAS C. CRAVEN, Department of Mathematics, University of Hawaii, Honolulu, HI 96822, Hawaii e-mail: tom@math.hawaii.edu}

\author{
TARA L. SMITH, Department of Mathematical Sciences, University of Cincinnati, \\ Cincinnati, OH 45221-0025, Ohio \\ e-mail: tara.smith@uc.edu
}

